\title{
Antiproliferative and proapoptotic activity of GUT-70 mediated through potent inhibition of Hsp90 in mantle cell lymphoma
}

\author{
L Jin ${ }^{1,2}$, Y Tabe ${ }^{*, 1}$, S Kimura ${ }^{3}$, Y Zhou', J Kuroda ${ }^{4}$, H Asou ${ }^{5}$, T Inaba ${ }^{5}$, M Konopleva $^{6}$, M Andreeff ${ }^{7}$ and T Miida' \\ 'Department of Clinical Laboratory Medicine, Juntendo University School of Medicine, 2-I I Hongo, Bunkyo-ku, Tokyo II 3-842 I, Japan; '2 Sportology \\ Center, Juntendo University School of Medicine, 2-1-I Hongo, Bunkyo-ku, Tokyo II 3-842 I, Japan; ${ }^{3}$ Division of Hematology, Respiratory Medicine and \\ Oncology, Department of Internal Medicine, Faculty of Medicine, Saga University, 5- I-I Nabeshima, Saga 849-850 I, Japan; ${ }^{4}$ Division of Hematology and \\ Oncology, Department of Medicine, Kyoto Prefectural University of Medicine, 465 Kajii-cho, Kamigyo-ku, Kyoto 602-8566, Japan; ${ }^{5}$ Department of \\ Molecular Oncology and Leukemia Program Project, Research Institute for Radiation Biology \& Medicine, Hiroshima University, I-2-3 Kasumi, Minami-ku, \\ Hiroshima 734-8553, Japan; 'Department of Leukemia, The University of Texas MD Anderson Cancer Center, 1515 Holcombe Blvd., Houston, \\ TX 77030, USA; ${ }^{7}$ Department of Stem Cell Transplantation and Cellular Therapy, The University of Texas MD Anderson Cancer Center, 15 I 5 Holcombe \\ Blvd., Houston, TX 77030, USA
}

BACKGROUND: Mantle cell lymphoma (MCL) is an aggressive B-cell lymphoma with poor prognosis, requiring novel anticancer strategies.

METHODS: Mantle cell lymphoma cell lines with known p53 status were treated with GUT-70, a tricyclic coumarin derived from Calophyllum brasiliense, and the biological and biochemical consequences of GUT-70 were studied.

RESULTS: GUT-70 markedly reduced cell proliferation/viability through $\mathrm{G}_{1}$ cell cycle arrest and increased apoptosis, with greater sensitivity in mutant (mt)-p53-expressing MCL cells than in wild-type (wt)-p53-bearing cells. Mechanistically, GUT-70 showed binding affinity to heat-shock protein 90 (Hsp90) and ubiquitin-dependent proteasomal degradation of Hsp90 client proteins, including cyclin DI, Raf-I, Akt, and mt-p53. Depletion of constitutively overexpressed cyclin DI by GUT-70 was accompanied by p27 accumulation and decreased Rb phosphorylation. GUT-70 induced mitochondrial apoptosis with Noxa upregulation and Mcl-I downregulation in mt-p53 cells, but Mcl-I accumulation in wt-p53 cells. Noxa and Mcl-I were coimmunoprecipitated, and activated BAK. Treatment with a combination of GUT-70 and bortezomib or doxorubicin had synergistic antiproliferative effects in MCL cells that were independent of p53 status.

CONCLUSION: GUT-70 has pronounced antiproliferative effects in MCL with mt-p53, a known negative prognostic factor for MCL, through Hsp90 inhibition. These findings suggest that GUT-70 has potential utility for the treatment of MCL.

British Journal of Cancer (201 I) 104, 91-100. doi:I0.1038/sj.bjc.6606007 www.bjcancer.com

Published online 7 December 2010

(c) 20II Cancer Research UK

Keywords: GUT-70; mantle cell lymphoma; apoptosis; p53; Hsp90; coumarin

Mantle cell lymphoma (MCL) is characterised by an aggressive clinical course, with rapid relapse after an initial response or primary resistance to standard chemotherapy (Jares et al, 2007). The $\mathrm{t}(11,14)(\mathrm{q} 13 ; 32)$ translocation of MCL leads to overexpression of cyclin D1, which is believed to be associated with oncogenesis by causing instability of the $\mathrm{G}_{1} / \mathrm{S}$ checkpoint through promotion of cyclin-dependent kinase activity and through sequestration of the Cip/Kip family of cyclin-dependent kinase inhibitors (Sherr and Roberts, 1999; Quintanilla-Martinez et al, 2003). These activities facilitate phosphorylation and inactivation of the retinoblastoma (Rb) $\mathrm{G}_{1} / \mathrm{S}$ checkpoint protein, resulting in cell cycle progression. It has been demonstrated, however, that overexpression of cyclin D1 itself is not sufficient for development of MCL, suggesting that additional genetic events might be necessary for oncogenesis (Bodrug et al, 1994), particularly as apoptosis-related genes such

*Correspondence: Dr Y Tabe; E-mail: tabe@juntendo.ac.jp Revised 6 October 2010; accepted 22 October 2010; published online 7 December 2010 as $p 53, I N K 4 a / A R F$, and $A T M$ are dysregulated in MCL (Fernàndez et al, 2005; Greiner et al, 2006).

In MCL, mutation/overexpression of $p 53$ is reported as an adverse prognostic indicator (Jares et al, 2007). As many of the antitumour effects mediated by chemotherapeutic agents depend on a p53-related pathway, resistance to chemotherapy often develops through impaired p53 signalling (Döhner et al, 1995). The 26S proteasome inhibitor bortezomib retains activity in $p 53-$ mutant (mt-p53) cells and has demonstrated single-agent efficacy in relapsed or refractory MCL, which is, however, based mainly on prolonged response rather than on an increase in ultimate survival rate (Goy et al, 2009).

Therefore, development of novel compounds that target $p 53$ independent signalling pathways is of considerable interest in the treatment of this disease.

We have reported that the newly discovered anticancer agent GUT-70, a natural product derived from the stem bark of Calophyllum brasiliense, demonstrated cytotoxic efficacy in human leukaemic cells (Kimura et al, 2005). GUT-70 (Figure 1), characterised as a tricyclic coumarin with the formula 
5-methoxy-2,2-dimethyl-6-(2-methyl-1-oxo-2-butenyl) -10-propyl$2 \mathrm{H}, 8 \mathrm{H}$-benzo[ $\left.1,2-b ; 3,4-b^{\prime}\right]$ dipyran-8-one $\left(\mathrm{C}_{23} \mathrm{H}_{26} \mathrm{O}_{5}\right)$, significantly inhibited leukaemic cell growth with a median inhibitory concentration $\left(\mathrm{IC}_{50}\right)$ of $2-5 \mu \mathrm{M}$ without repressing colony formation by normal haematopoietic progenitors or proliferation of normal human hepatocytes at concentrations up to $30 \mu \mathrm{M}$ (Kimura et al, 2005).

Coumarin antibiotics have been reported to bind the newly discovered C-terminal ATP binding site of $90 \mathrm{kDa}$ heat-shock protein (Hsp90), a molecular chaperone responsible for the folding and conformational maintenance of client proteins (Marcu et al, 2000; Issacs et al, 2003; Pratt and Toft, 2003; Donnelly et al, 2008). Hsp90 inhibition results in degradation of misfolded Hsp90 clients through ubiquitination, followed by proteasome-mediated hydrolysis (Zhang et al, 2004). As many of the Hsp90 client proteins contribute to cancer cell proliferation, Hsp90 has emerged as a promising target for cancer chemotherapy (Issacs et al, 2003; Donnelly et al, 2008).

In this study, GUT-70 demonstrated antiproliferative and proapoptotic activities with more prominent efficacy in $\mathrm{mt}-\mathrm{p} 53$ bearing MCL cells than in those with wild-type (wt) p53. GUT-70 showed binding affinity to Hsp90, and reduced expression of Hsp90 client proteins such as mt-p53, Raf-1, cyclin D1, and Akt. The intrinsic apoptotic pathway was activated by GUT-70 through upregulation of Noxa and BAK activation. The combination of GUT-70 with bortezomib or doxorubicin yielded synergistic antiproliferative effects independent of $p 53$ status. These findings indicate possible efficacy and a rationale for further exploration of GUT-70 as a new therapeutic strategy for MCL.

\section{MATERIALS AND METHODS}

\section{Cell lines and culture conditions}

Four MCL cell lines were used in this study: JVM-2 (Melo et al, 1986), Granta 519 (Jadayel et al, 1997) and MINO (Lai et al, 2002) were kindly provided by Dr M Raffeld, and Jeko-1 (Raynaud et al, 1993) was a gift from Dr M Seto. Granta 519 and JVM-2 express wt- $p 53$, whereas Jeko- 1 and MINO express $p 53$ mutations (Jeko-1, loss of p53 expression; MINO, mutation at codon 147 (valine $\rightarrow$ glycine)) (Raynaud et al, 1993; Lai et al, 2002). JVM-2, Jeko-1 and MINO were cultured in RPMI 1640 medium containing $15 \%$ fetal bovine serum (FBS) and 1\% penicillin/streptomycin. Granta 519 was grown in Dulbecco's modified Eagle's medium (DMEM) supplemented with $15 \%$ FBS. Cells were first acclimated in RPMI 1640 or DMEM containing 5\% FBS for $16 \mathrm{~h}$ before exposure to GUT-70 (Nippon Shinyaku, Kyoto, Japan) (Kimura et al, 2005). Control cells were treated with an equivalent amount of dimethyl sulphoxide (DMSO). Doxorubicin was obtained from Sigma
(St Louis, MO, USA) and bortezomib was provided by Millennium (Cambridge, MA, USA). Human osteosarcoma cell line U2OS transfected with the histone cluster $1(H 2 B K)$ and enhanced green fluorescent protein (EGFP) genes, U2OS-H2BK-EGFP, was grown in DMEM supplemented with $10 \%$ FBS and used for morphological observation. U2OS expresses wt-p53 (Flørenes et al, 1994).

\section{Cell viability/proliferation assay}

Cell viability was assessed by the Trypan blue dye exclusion method, and cell proliferation was determined by the CellTiter 96 AQueous One Solution Cell Proliferation Assay (MTS; Promega, Madison, WI, USA).

\section{Apoptosis analysis}

Apoptotic cell death was evaluated through annexin $\mathrm{V}$ (Roche Diagnostic, Indianapolis, IN, USA) and propidium iodide (PI) positivities by a FACScan flow cytometer and Cell Quest software (Becton Dickinson Immunocytometry Systems, San Jose, CA, USA). The extent of drug-specific apoptosis was assessed by the following formula: $\%$ specific apoptosis $=($ test - control $) \times 100 /$ (100-control).

\section{Flow cytometric analysis of cell cycle and BAK activation}

Cell cycle distribution was determined by flow cytometric analysis of PI-stained nuclei. DNA content was determined by FACScan flow cytometer and CellQuest software. BAK activation was analyzed as previously described (Samraj et al, 2006). Briefly, cells were fixed and permeabilized using the DAKO IntraStain kit (DakoCytomation, Glostrup, Denmark) according to the manufacturer's instructions. Cells were then stained with conformationspecific monoclonal antibody against BAK (y164; Abcam, Cambridge, MA, USA) or isotype-matched control antibody for $30 \mathrm{~min}$ at room temperature, followed by incubation with Alexafluor 488-labeled chicken anti-rabbit secondary antibody (Molecular Probes, Eugene, OR, USA) for $30 \mathrm{~min}$ on ice in the dark. After the washing step, conformational change of BAK was analyzed by a FACScan flow cytometer.

\section{Western blot analysis and immunoprecipitation}

Cells were solubilised in lysis buffer (phosphate-buffered saline solution (PBS), $1 \times$ cell lysis buffer (Cell Signaling Technology, Danvers, MA, USA), $1 \times$ protease inhibitor (Roche), and $1 \times$ phosphatase inhibitor cocktails I and II (Calbiochem, San Diego, CA, USA)), and incubated for $30 \mathrm{~min}$ on ice. Total protein $(20 \mu \mathrm{g})$ was separated by sodium dodecyl sulphate polyacrylamide gel electrophoresis (SDS-PAGE), immunoblotted with appropriate antibodies, and reacted with enhanced chemiluminescence reagent (Amersham Biosciences, Piscataway, NJ, USA); signals were detected by a luminescent image analyser (LAS-1000 plus; Fujifilm, Tokyo, Japan). The anti- $\alpha$-tubulin or anti- $\beta$-actin blot was used in parallel as a loading control. For immunoblotting, the following antibodies were used: $\mathrm{p} 21^{\mathrm{Cip} 1 / \mathrm{WAF} 1}, \mathrm{p} 27^{\mathrm{KIP} 1}$, and $\mathrm{Mcl}-1$ (BD-Pharmingen, San Diego, CA, USA); p53 (DO-7; Dako, Carpinteria, CA, USA); Noxa (Calbiochem); $\alpha$-tubulin (SigmaAldrich, St Louis, MO, USA); Puma (Upstate Biotechnology, Lake Placid, NY, USA); LC-3 (MBL, Nagoya, Japan); ubiquitin (Santa Cruz Biotechnology, Santa Cruz, CA, USA); and Hsp70, c-Raf, Akt, ERK1/2, phosphorylated-ERK1/2 Thr202/Tyr204(p-ERK1/2), cyclin D1, phosphorylated $\mathrm{Rb}^{\mathrm{Ser} 780}(\mathrm{p}-\mathrm{Rb}), \mathrm{Bim}, \mathrm{BAK}$, cleaved caspase-9, cleaved caspase-3, $\beta$-actin, and horseradish peroxidase-linked antimouse and anti-rabbit IgG (all from Cell Signaling Technology). Protein lysates were subjected to immunoprecipitation using antiMcl-1 (Santa Cruz Biotechnology). 


\section{Hsp90 binding assay}

The Hsp90 $\alpha$ inhibitor screening assay kit with $\mathrm{Hsp} 90 \alpha$ recombinant enzyme and fluorescein isothiocyanate (FITC)-labelled geldanamycin was used (BPS Bioscience, San Diego, CA, USA). The competition of fluorescence-labelled geldanamycin for binding to purified recombinant Hsp $90 \alpha$ was measured by Flex Station 3 (Molecular Devices, Sunnyvale, CA, USA).

\section{Morphological observation}

U2OS-H2BK-EGFP cells $\left(2.0 \times 10^{5}\right.$ per $\left.\mathrm{ml}\right)$ were cultured in a $35-\mathrm{mm}$ dish and treated with $5 \mu \mathrm{M}$ GUT-70 or DMSO only. Each dish was placed on the stage of a light microscope equipped with a digital camera (BZ-8000; Keyence, Osaka, Japan) at $37^{\circ} \mathrm{C}$ under a humidified atmosphere of $5 \% \mathrm{CO}_{2}$. Video images were collected over the period from 12 to $48 \mathrm{~h}$ after treatment.

\section{mRNA quantification by real-time reverse-transcriptase PCR (RT - PCR)}

Total RNAs were extracted from cells with the RNeasy Mini Kit (Qiagen, Hilden, Germany). First-strand cDNA synthesis was performed with oligo(dT) as primer (Superscript II System; Invitrogen, Carlsbad, CA, USA). Real-time reverse-transcriptase PCR was performed by the Model 7500 Real-time PCR System (Applied Biosystems, Foster City, CA, USA). Expression of Noxa and GAPDH mRNA was detected by TaqMan Gene Expression Assays (Noxa: Hs00560402_m1, GAPDH: Hs99999905_m1; Applied Biosystems). The PCR cycle number that generated the first

A

JVM-2

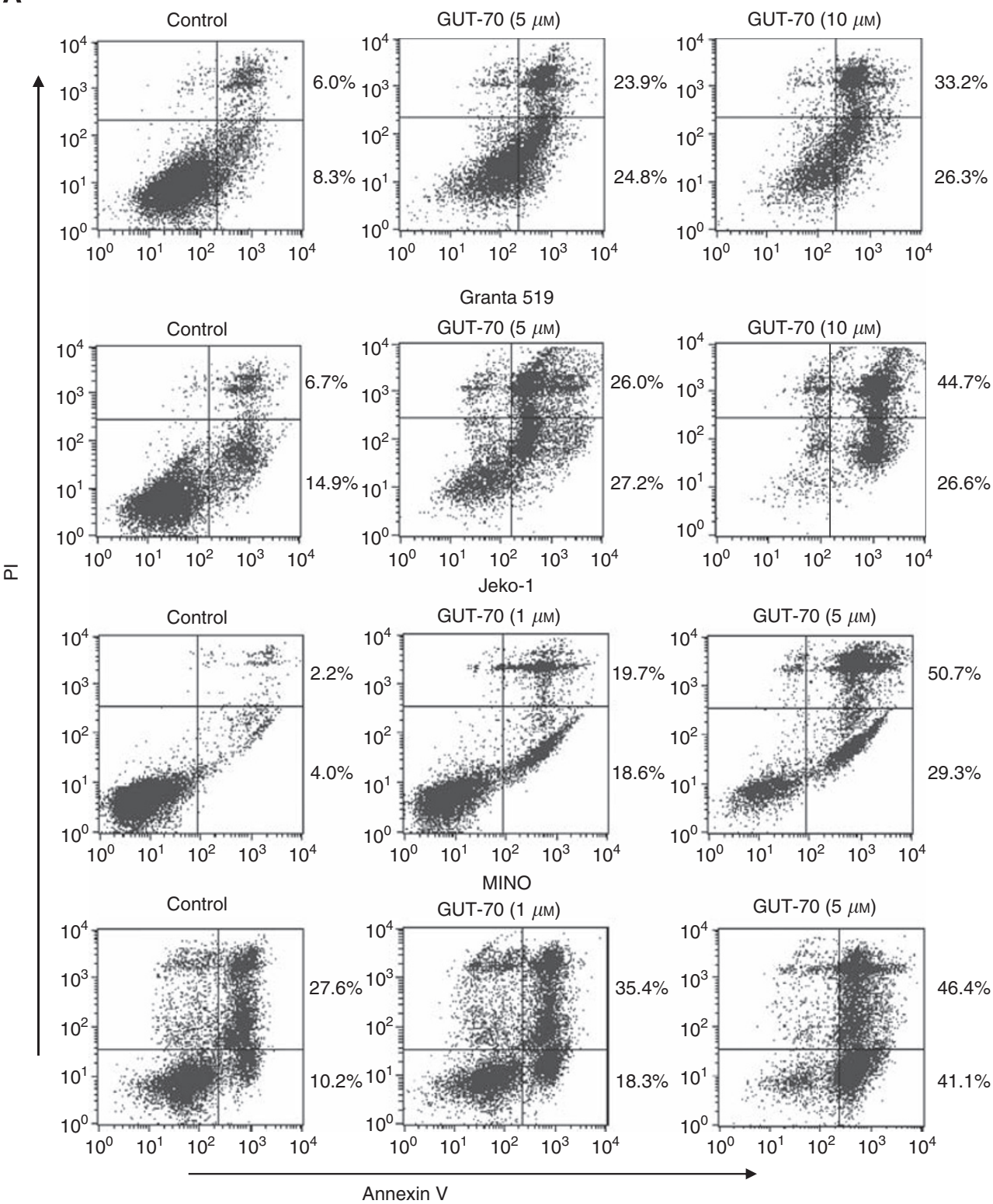

Figure 2 GUT-70-induced cell growth inhibition, apoptosis, and cell cycle arrest in MCL. (A) JVM-2, Granta 5I9, Jeko-I, and MINO cells were treated with the indicated concentrations of GUT-70 for $48 \mathrm{~h}$, and the percentages of apoptotic cells were quantified by annexin V/PI staining. (B) Representative flow cytometric histograms of Pl-treated cells after $24 \mathrm{~h}$ of GUT-70 treatment at indicated concentrations. The percentages of $\mathrm{G}_{0} / \mathrm{G}_{1-}, \mathrm{S}_{-}$and $\mathrm{G}_{2} / \mathrm{M}_{-}$-phase cells were assessed in total viable cells (bar graphs). 
B

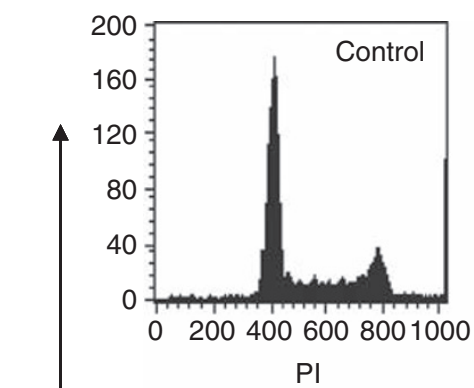

JVM-2
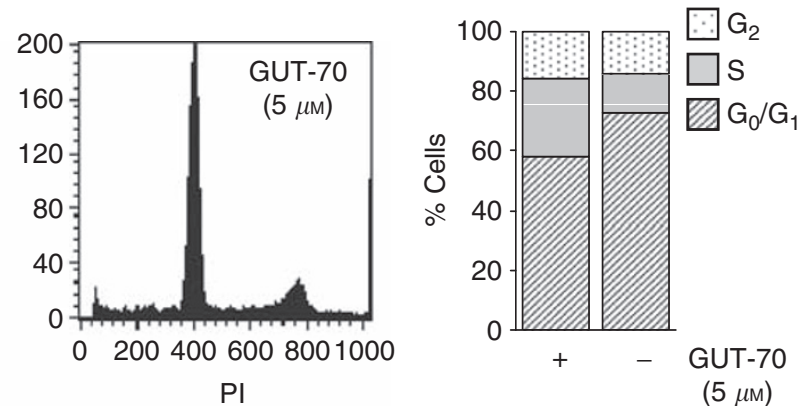

Granta 519
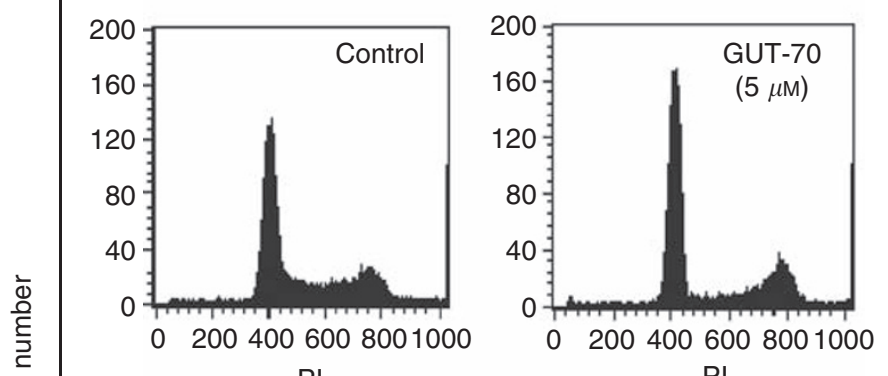

$\mathrm{PI}$

Jeko-1
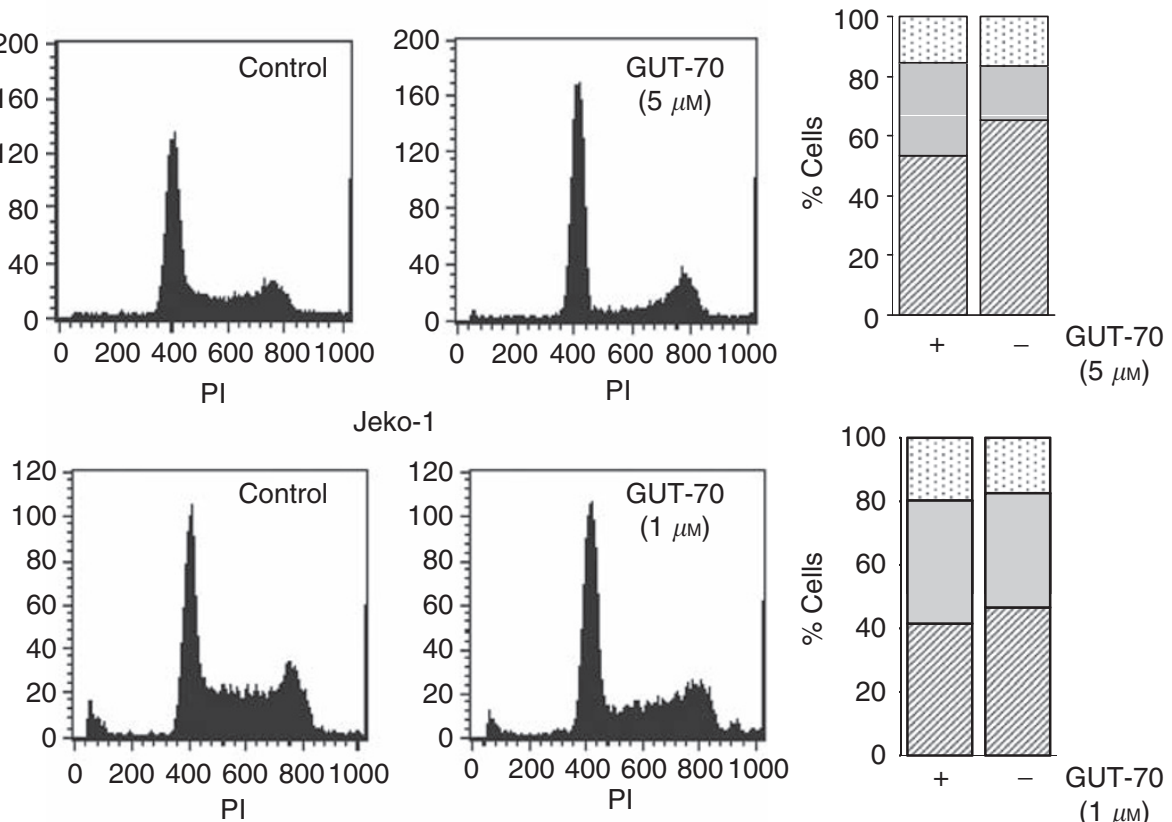

MINO

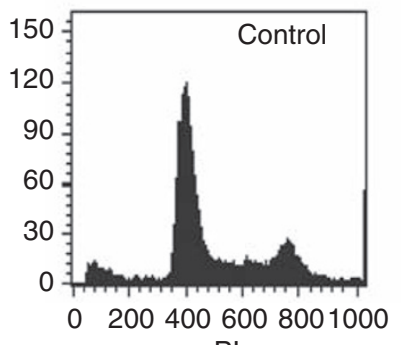

$\mathrm{PI}$

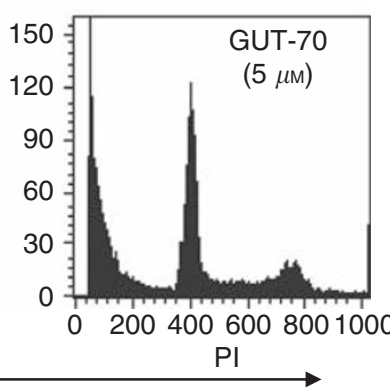

DNA content

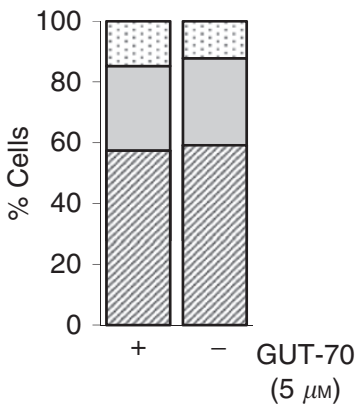

Figure 2 Continued.

fluorescence signal above a threshold value (the threshold cycle; $C_{\mathrm{t}}$ ) was determined. The abundance of each transcript of Noxa relative to that of $G A P D H$ was calculated as follows: relative expression $=100 \times 2 \exp \left[-\Delta C_{\mathrm{t}}\right]$, where $\Delta C_{\mathrm{t}}$ is the mean $C_{\mathrm{t}}$ of the transcript of interest minus the mean $C_{\mathrm{t}}$ of the transcript for $G A P D H$. The $C_{\mathrm{t}}$ data from duplicate PCRs were averaged for calculation of relative expression.

\section{Statistical analysis}

Cytotoxicity was assessed by the Chou-Talalay method (Chou and Talalay, 1984) using Calcusyn software (Biosoft, Cambridge, UK). The combination index $(\mathrm{CI})$ values indicate degree of synergism: strong synergism $(0.3-0.7)$, moderate synergism $(0.7-0.85)$, and slight synergism $(0.85-0.9)$.

\section{RESULTS}

\section{GUT-70 induces apoptosis and cell cycle arrest in MCL cells}

Treatment with GUT-70 (Figure 1) for $48 \mathrm{~h}$ resulted in dosedependent cell growth inhibition detected by MTS cell proliferation assay ( IC $_{50}$ : Granta 519, 6. $3 \mu \mathrm{M}$; JVM-2, $4.5 \mu \mathrm{M}$; Jeko-1, $1.7 \mu \mathrm{M}$; MINO, $1.5 \mu \mathrm{M}$ ).

To determine whether the inhibition of cell growth by GUT-70 was associated with apoptosis and/or cell cycle arrest, we 
A

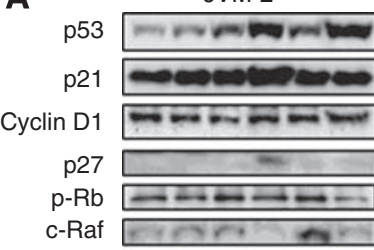

Akt mrarsart

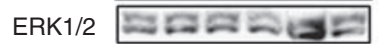

p-ERK1/2

Hsp70

$\alpha$-Tubulin

GUT-70

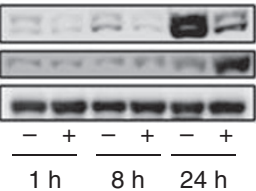

Granta 519
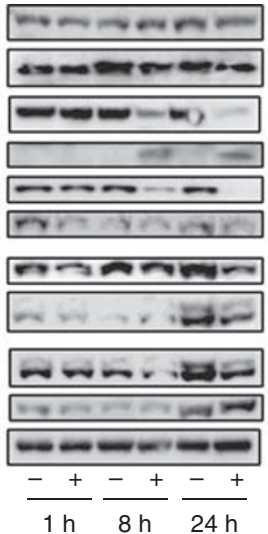
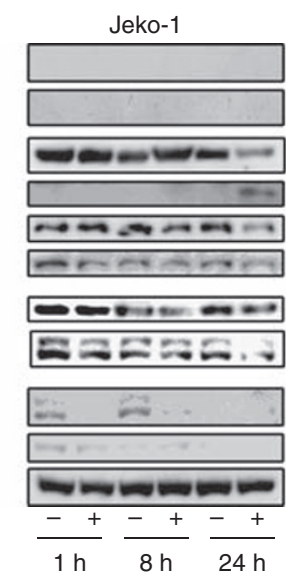

MINO
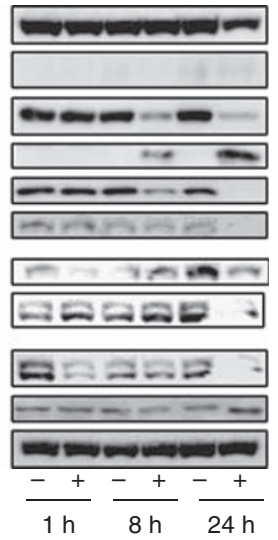

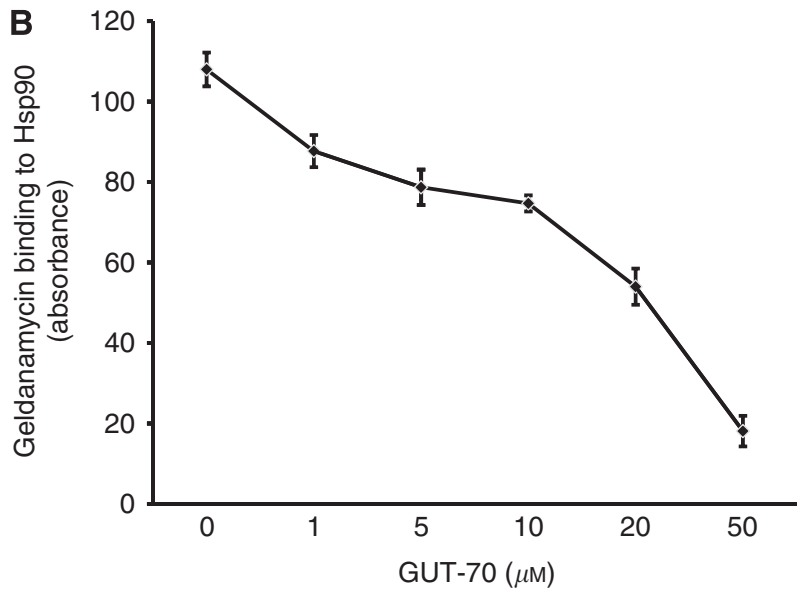

C

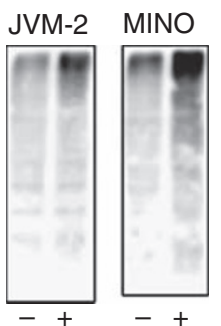

D

GUT-70

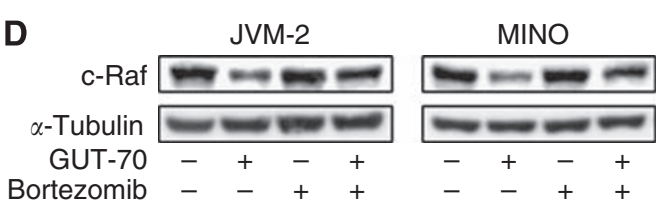

Figure 3 (A) GUT-70 effects on Hsp90 client proteins. MCL cells were treated with GUT-70 (JVM-2, $5 \mu \mathrm{M}$; Granta 5 I 9, $5 \mu \mathrm{M}$; Jeko- I, I $\mu \mathrm{M}$; MINO, $5 \mu \mathrm{M}$ ) for indicated times. Cells were subjected to lysis and analysed by western blot. Western blot images are representative results from three independent experiments. (B) GUT-70 competitively inhibited geldanamycin binding to the Hsp90 $\alpha$ subunit. The competition of GUT-70 with FITC-labelled geldanamycin for binding to purified recombinant Hsp90 $\alpha$ was measured. Fluorescence was measured at $\lambda e x 485 \mathrm{~nm}$ and at $\lambda \mathrm{em} 525 \mathrm{~nm}$. Results shown are means \pm s.d. from three independent experiments. (C) GUT-70 induced protein ubiquitination. JVM-2 and MINO cells were treated with $5 \mu$ M GUT-70 for $24 \mathrm{~h}$, subjected to lysis, and immunoblotted for ubiquitin. Representative results are shown from three independent experiments. (D) Proteasome inhibitor bortezomib prevented GUT-70-mediated decreased expression of c-Raf. JVM-2 and MINO cells were treated with $5 \mu$ M GUT-70 and/or I0 nM bortezomib for $24 \mathrm{~h}$, subjected to lysis, and immunoblotted for c-Raf. Western blot images are representative results from three independent experiments.

conducted flow cytometric analysis of annexin V/PI-stained and PI-stained nuclei. As shown in Figure $2 \mathrm{~A}, 48 \mathrm{~h}$ of GUT-70 treatment induced dose-dependent increases of annexin $\mathrm{V}$ positivity in all cell lines; this effect was more pronounced in mt-p53-bearing Jeko-1 and MINO cells than in wt-p53-bearing Granta 519 and JVM-2 cells (specific apoptosis by $5 \mu$ M GUT-70: $40.3 \%$ for Granta $519,40.1 \%$ for JVM-2, $78.8 \%$ for Jeko-1, $79.9 \%$ for MINO). The PI cell cycle histograms further demonstrated that GUT-70 increased the sub- $G_{1}$ fraction in a time-dependent manner at a lower dose for mt- $p 53$ cells than for wt- $p 53$ cells; sub- $G_{1}$ fractions at 24 and $48 \mathrm{~h}$ were 4.6 and $10.7 \%$ for Granta $519(5 \mu \mathrm{M}$ GUT-70), 14.8 and $34.7 \%$ for JVM-2 $(5 \mu \mathrm{M}), 5.2$ and $19.3 \%$ for Jeko$1(1 \mu \mathrm{M})$, and 12.0 and $34.9 \%$ for MINO $(1 \mu \mathrm{M})$. Whereas GUT-70 impeded $\mathrm{G}_{1}-\mathrm{S}$ cell cycle progression in JVM-2 and Granta 519 cells, $\mathrm{G}_{1}-\mathrm{S}$ arrest was minimal in MINO and Jeko-1 cells (Figure $2 \mathrm{~B}$ ). These data suggest that GUT-70-induced cell growth inhibition resulted in part from cell cycle arrest at the $G_{0} / G_{1}$ checkpoint and in part from apoptosis induction.

\section{GUT-70 downregulates mutated p53 and cyclin D1 and accumulates p27}

We next investigated changes in cell cycle regulatory proteins associated with GUT-70 treatment. As shown in Figure 3A, GUT-70 induced p53/p21 accumulation in JVM-2 cells, but did not increase p53/p21 expression in Granta 519 cells. In Jeko-1 cells, basal p53/p21 expression was not detectable and was unaffected by GUT-70. Notably, expression of the overexpressed mt-p53 protein was reduced in MINO cells by $24 \mathrm{~h}$ exposure to GUT-70, without detectable p21 expression. The expression level of p27 was upregulated by GUT-70, irrespective of p53 status. GUT-70 diminished the highly expressed cyclin D1 in all tested MCL cells except JVM-2, and resulted in substantial decreases in $\mathrm{Rb}$ phosphorylation in all tested cells (Figure 3 ).

\section{GUT-70 induces degradation of Hsp90 substrate proteins}

The coumarin antibiotics have been reported to bind to Hsp90 (Marcu et al, 2000). To investigate whether GUT-70 has binding affinity for Hsp90, a competitive binding assay was performed using geldanamycin, a well-characterised ATP competitive inhibitor (Gooljarsingh et al, 2006). GUT-70 demonstrated dosedependent inhibition of geldanamycin binding to Hsp90, which indicated the binding activity of GUT-70 to Hsp90 (Figure 3B). The degradation by GUT-70 of Hsp90 client proteins, such as Raf-1 and its downstream ERK1/2 and phospho ERK1/2, as well as Akt (Pratt and Toft, 2003; Zhang et al, 2004, 2005), was detected by 
A

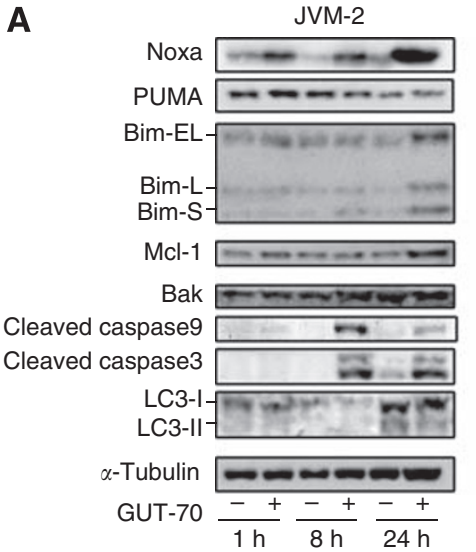

Granta 519

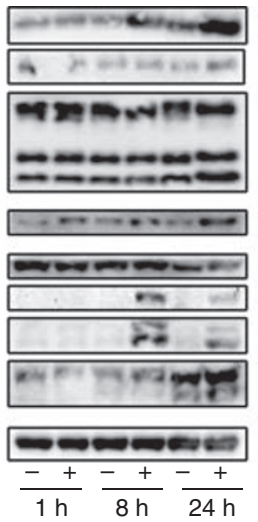

Jeko-1

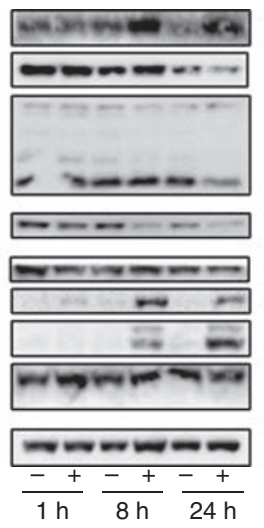

MINO

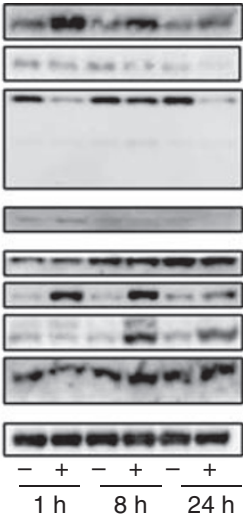

B

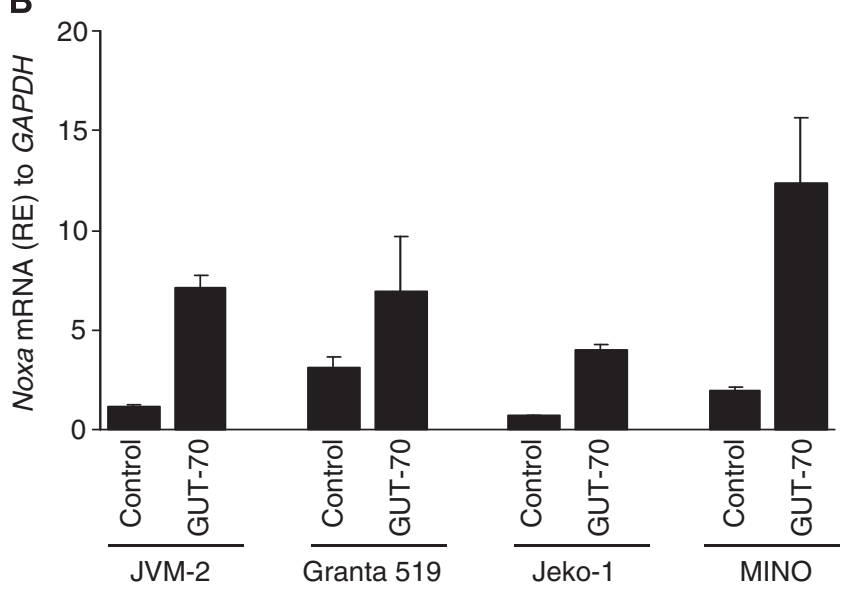

C

믐

IP: Mcl-1

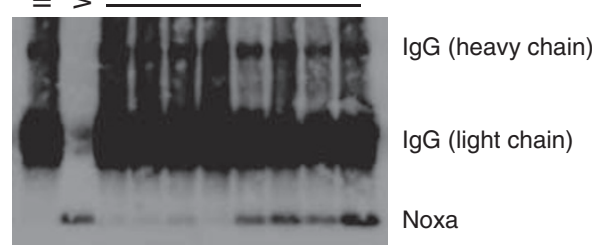

GUT-70
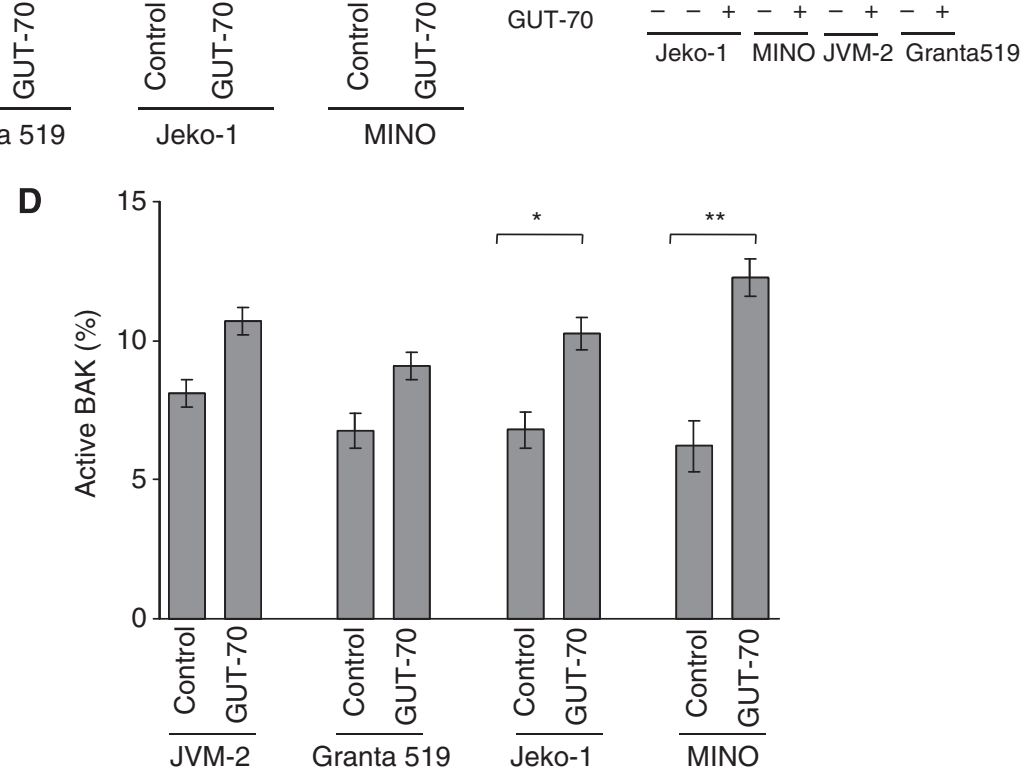

Figure 4 Modulation of apoptosis-related protein levels by GUT-70. MCL cells were treated with GUT-70 (JVM-2, $5 \mu \mathrm{M}$; Granta 5I 9, $5 \mu \mathrm{M}$; Jeko- I, I $\mu$ M; $\mathrm{MINO}, 5 \mu \mathrm{M}$ ) for the indicated times. (A) Cells were subjected to lysis, then apoptosis-related proteins and macroautophagy marker LC3 were analysed by western blot. Western blot images are representative results from three independent experiments. (B) Cells were harvested after $18 \mathrm{~h}$ treatment with GUT-70, and Noxa mRNA expression levels were detected by TaqMan RT-PCR analysis. The abundance of transcripts of Noxa relative to GAPDH transcripts was determined as described in Materials and Methods. Graphs show the representative data from two independent experiments with similar results. (C) Cells were treated with GUT-70 for $24 \mathrm{~h}$, and Mcl-I immunoprecipitation was performed as described in Materials and Methods. Total extracts were analysed by western blotting for Noxa. Western blot images are representative results from three independent experiments. (D) Cells were treated with GUT-70 for $24 \mathrm{~h}$, then conformational changes in BAK were measured by intracellular flow cytometry as described in Materials and methods. To block the caspase activation-mediated conformational changes of BAK, cells were preincubated for I $\mathrm{h}$ with $100 \mu \mathrm{M}$ Z-VAD-FMK. Data represent duplicate experiments. $* P<0.01$; $* * P<0.05$. RE, relative expression.

western blot analysis in all tested MCL cells (Figure 3A). Cyclin D1 and $\mathrm{mt}-\mathrm{p} 53$, the expression of which was repressed by GUT-70, are known substrate proteins of Hsp90 (Zhang et al, 2004; Muller et al, 2008). Furthermore, GUT-70 increased expression of Hsp70, a marker of Hsp90 inhibition (Elo et al, 2005; Bao et al, 2009), in Granta 519, JVM-2, and MINO cells. In Jeko-1 cells, however, Hsp70 was detected at a level insufficient to be reliable as a marker without further induction by GUT-70 (Figure 3A). 
A
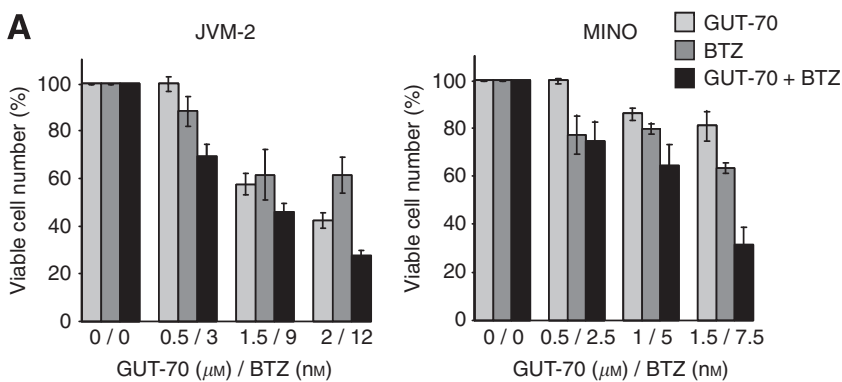

B
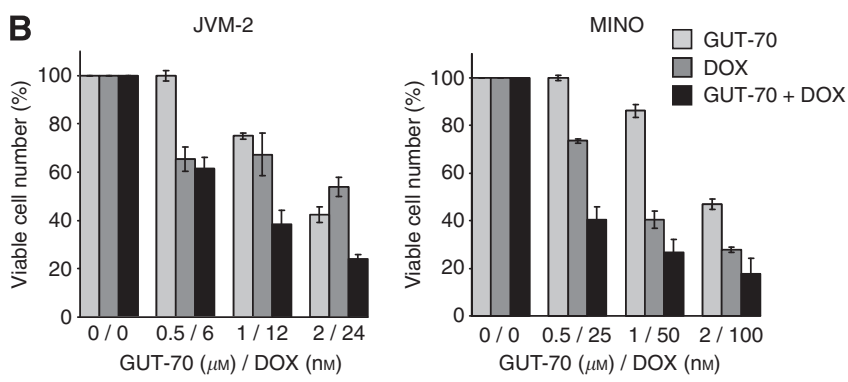

Figure 5 Synergistic interaction between GUT-70 and bortezomib or doxorubicin in MCL cells. JVM2 and MINO cells were cultured in the presence of escalating doses of GUT-70 and bortezomib (BTZ) (A) or GUT-70 and doxorubicin (DOX) (B) at a fixed ratio. After $48 \mathrm{~h}$, viable and dead cells were identified using the Trypan blue dye exclusion method. Results are expressed as means \pm s.d. percentage of viable cell numbers in control cells.

As the client proteins of Hsp90 chaperone molecule become misfolded and ubiquitinated by Hsp90 inhibition, and are then downregulated by proteasomal degradation (Issacs et al, 2003; Zhang et al, 2004), we next tried to determine whether GUT-70 induces protein ubiquitination followed by proteasomal degradation in wt-p53-expressing JVM-2 and mt-p53-expressing MINO cells. As expected, GUT-70 treatment elevated the level of protein ubiquitination (Figure 3C); subsequent treatment with proteasome inhibitor bortezomib prevented degradation of c-Raf by GUT-70 (Figure 3D). Taken together, these data indicate the interaction of GUT-70 with Hsp90 and the destabilisation of Hsp90 client proteins by GUT-70.

\section{Effect of GUT-70 on apoptosis-related proteins}

To characterise the mechanism of GUT-70-induced cell death in MCL cells, we analysed the expression of apoptosis-related Bcl-2 family proteins, the BH3-only proteins Noxa, Puma, and Bim, and the other Bcl-2 family proteins, Mcl-1 and BAK, in MCL cell lines. Results show that GUT-70 induced substantial accumulation of Noxa but not of Puma (Figure 4A). Mutant-p53-bearing MCL cells demonstrated earlier Noxa induction than wt-p53 cells; peak induction of Noxa was observed after $1 \mathrm{~h}$ of GUT-70 treatment in MINO, after $8 \mathrm{~h}$ in Jeko-1, and after $24 \mathrm{~h}$ in JVM-2 and Granta 519 cells. GUT-70 induced upregulation of Noxa mRNA levels in all tested cells (Figure 4B).

After $24 \mathrm{~h}$ of GUT-70 treatment, levels of antiapoptotic protein Mcl-1 were increased in JVM-2 and Granta 519 cells, but decreased in Jeko-1 and MINO cells (Figure 4A). It is known that Noxa binds preferentially to Mcl-1 (Warr and Shore, 2008), triggers BAK or Bim release from $\mathrm{Mcl}-1$, and then starts the mitochondrial apoptotic pathway (Willis et al, 2005; Hauck et al, 2009). Concordantly, we detected coimmunoprecipitation between Noxa and Mcl-1 (Figure 4C) in JVM-2 and Granta 519 cells, both of which showed accumulation of Mcl-1 induced by GUT-70. Although total
BAK expression levels remained, consistently independent of GUT-70 treatment (Figure 4A), flow cytometric analysis revealed a pronounced increase of activated BAK in MINO, moderate activation in Jeko-1, and only slight activation in JVM-2 and Granta 519 cells after GUT-70 treatment (Figure 4D), activities that are in inverse relation to $\mathrm{Mcl}-1$ expression levels.

Proapoptotic $\mathrm{BH}-3$-only protein Bim was induced by GUT-70 at $24 \mathrm{~h}$ in JVM-2 cells but not in the other cell lines (Figure $4 \mathrm{~A}$ ).

\section{GUT-70 does not induce macroautophagy}

Increasing evidence indicates that autophagy is one of the important mechanisms of anticancer reagent-induced cell death (Tsujimoto and Shimizu, 2005). In mammals, three modes of autophagy have been identified: macroautophagy, microautophagy, and chaperone-mediated autophagy (Levine and Klionsky, 2004). To investigate the possibility that GUT-70 promotes macroautophagy, we examined the conversion of light chain 3 (LC3) from LC3-I to LC3-II, a marker of autophagosome formation (Kabeya et al, 2000). Whereas LC3-II was moderately induced by a low serum culture condition $(5 \%, 40 \mathrm{~h})$ in wt-p53-expressing JVM-2 and Granta 519 cells, there was no change in accumulation of LC3-II following further treatment with GUT-70 $(24 \mathrm{~h})$. In mtp53-bearing MINO and Jeko-1 cells, neither serum starvation nor GUT-70 treatment induced LC3-II accumulation (Figure 4A).

To assess the morphological changes induced by GUT-70, U2OS-H2BK-EGFP cells were sequentially photographed after exposure to GUT-70. Cells underwent morphological alterations, including cytoplasmic swelling and vacuolisation after $24 \mathrm{~h}$ of GUT-70 exposure (cellular oncosis), and cell death peaked at $36 \mathrm{~h}$ (secondary necrosis) (Supplementary Material 1 for Quick-Time movies) (Majno and Joris, 1995; Lemasters et al, 1998; Van Cruchten and Van Den Broeck, 2002).

\section{Combination of GUT-70 with bortezomib or doxorubicin has synergistic effects on MCL growth inhibition}

To determine whether GUT-70 potentiates the commonly used chemotherapeutic agents, we assessed the effects of combinations of GUT-70 with bortezomib, a selective inhibitor of the $26 \mathrm{~S}$ proteasome, or doxorubicin, a conventional chemodrug for MCL, on viability of wt- $p 53 \mathrm{JVM}-2$ and mt-p53 MINO cells. As shown in Figure $5 \mathrm{~A}$, both of these combination treatments had observable synergistic effects in both cell types $48 \mathrm{~h}$ after exposure. The averaged CI values of GUT-70/bortezomib treatment were 0.59 for JVM2 and 0.73 for MINO; for GUT-70/doxorubicin, 0.37 for JVM2 and 0.35 for MINO, indicating strong and moderate synergism, respectively.

\section{DISCUSSION}

The natural product-derived tricyclic coumarin GUT-70 exhibited single-agent antiproliferative and proapoptotic activities against MCL cell lines as a novel Hsp90 inhibitor. GUT-70's dosedependent inhibition of geldanamycin binding to Hsp90 $\alpha$ indicates that GUT-70 has direct binding activity to Hsp90, by which GUT70 induces conformational change in the Hsp90 molecule and interferes with its binding of geldanamycin. This finding agrees with that of previous studies showing that coumarin antibotic novobiocin binds to the Hsp90 C-terminal ATP binding site and affects the binding of geldanamycin at the Hsp90 N-terminal domain through close interaction between amino and carboxy termini in solution (Csermely et al, 1998; Hartson et al, 1999; Marcu et al, 2000; Donnelly et al, 2008). GUT-70 induced depletion of Hsp90 client proteins mt-p53, Raf-1, cyclin D1, and Akt, and increased Hsp70, a marker of Hsp90 inhibition; these findings, along with the ubiquitin-dependent proteasomal degradation of 
Hsp90 client proteins, suggest that GUT-70 functions as an Hsp90 inhibitor.

It is important that mt-p53-expressing MCL cells were more sensitive to GUT-70-induced apoptosis than wt-p53-bearing MCL cells. In mt- $p 53$ cells, prominent GUT-70-induced apoptosis was accompanied by minimal cell cycle arrest, which is consistent with a previous report of $\mathrm{G}_{2} / \mathrm{M}$ checkpoint abrogation in $\mathrm{p} 53 / \mathrm{p} 21$ impaired cells through downregulation of Chk1 and Weel by Hsp90 inhibitor that resulted in premature mitotic entry and mitotic death (Tse et al, 2009).

Furthermore, GUT-70 induced the most pronounced apoptosis in MINO cells in which GUT-70 treatment depleted overexpressed mt-p53. mt-p53 is known to confer the additional 'gain of function' as the transcription regulator. Transcriptional activation by mt-p53 has been reported for MDR1 (Sampath et al, 2006), c-MYC (Frazier et al, 1998), or GRO1 (Yan and Chen, 2009), resulting in cell proliferation, antiapoptosis, and tumourigenicity (Blandino et al, 1999). GUT-70-induced degradation of mt-p53 may successfully repress these oncogenic transcriptional activations.

Another important finding of this study is the prominent p53-independent Noxa upregulation by GUT-70. Whereas Noxa had been proposed to be a critical mediator of $p 53$-dependent apoptosis (Oda et al, 2000), p53-independent upregulation of Noxa has been described in MCL and B-cell chronic lymphocytic leukaemia (Pérez-Galán et al, 2006; Smit et al, 2007). Furthermore, GUT-70 induced Noxa protein accumulation extremely early $(1 \mathrm{~h})$ in mt-p53-bearing MINO cells, indicating independence from transcriptional gene induction. Recently, Noxa degradation by direct interaction with a spliced isoform of the Kruppel-like tumour suppressor (KLF6-SV1) (Difeo et al, 2009), or by posttranscriptional stabilisation/destabilisation of Bim mRNA (Matsui et al, 2007), has been reported. Our findings indicate the possibility of posttranscriptional Noxa stabilisation by GUT-70, which requires further elucidation.

The preferred binding partner of Noxa is the multidomain antiapoptotic Bcl-2 family member Mcl-1. In response to apoptotic stimuli, Noxa binds to Mcl-1, which ultimately leads to activation of BAK by releasing BAK from the BAK-Mcl-1 complex, and triggers BAK-mediated cell death (Chen et al, 2005; Warr and Shore, 2008) (Figure S, Supplementary Material 2 (Kuroda and Kimura, 2007)). The balance between Noxa and Mcl-1 is proposed to determine cell fate as death versus survival (Mei et al, 2007). GUT-70-induced BAK activation and sequential apoptosis were associated with Mcl-1 accumulation levels; high levels in less-sensitive wt-p53 cells and low levels in highly sensitive mt-p53 cells were consistent with previous reports (Pérez-Galán et al, 2006; Mei et al, 2007).

Autophagy is known to promote both autophagic cell death and cell survival (Kamitsuji et al, 2008). Although GUT-70 did not affect autophagosome formation, Hsp90 clients have been shown to be degraded through chaperone-mediated autophagy (Shen et al, 2009). The role of GUT-70 in induction of chaperonemediated autophagy requires further elucidation.

The observed morphological changes in GUT-70-treated cells (e.g., swelling cytoplasm) indicate cellular oncosis (Van Cruchten and Van Den Broeck, 2002), which shares certain mechanisms and alterations with apoptosis, such as loss of mitochondrial permeability and membrane potential (Lemasters et al, 1998).

Furthermore, our results demonstrate that GUT-70 can synergise the cytotoxic effects of the proteasome inhibitor bortezomib and the widely used genotoxic chemotherapeutic agent doxorubicin in MCL cells (Brody and Advani, 2006; Goy et al, 2009), regardless of p53 status. Previously, a combination of Hsp90 inhibitor geldanamycin and bortezomib was demonstrated to simultaneously disrupt $\mathrm{Hsp} 90$ and proteasome function, promote accumulation of ubiquitinated proteins, and enhance antitumour activity in human breast cancer cells (Mimnaugh et al, 2004, 2006). Whereas bortezomib induces longer-term remission (Goy et al, 2009), patients ultimately succumb to the poor clinical outcome, and there is a critical need to develop the most effective combination. The synergistic effects of GUT-70 and bortezomib may offer more efficacy and flexibility to the treatment of MCL with bortezomib. The antiproliferative effect of the combination of doxorubicin and GUT-70 was consistent with the previous findings for doxorubicin and Hsp-90 inhibitor 17-(dimethylaminoethylamino)-17-demethoxygeldamanycin (DMAG), which induced premature mitosis, followed by apoptosis, by bypassing the $G_{2} / M$ checkpoint in lymphoma cells (Robles et al, 2006). The synergy with doxorubicin suggests that addition of GUT-70 may allow reduction in the therapeutic dose of doxorubicin, which could potentially reduce its genotoxic side effects (Brody and Advani, 2006). A development of in vivo studies of these combination treatments for MCL is further required.

In conclusion, our results demonstrate that the novel anticancer agent GUT-70, a tricyclic coumarin, inhibits cell proliferation by depleting Hsp90 substrates cyclin D1, Akt, and Raf-1, and induces mitochondrial apoptotic cell death with upregulation of Noxa in MCL cells. Notably, these effects are substantially pronounced in MCL cells with mt-p53, a known negative prognostic factor for MCL. These findings suggest that GUT-70 has potential utility for the treatment of MCL.

\section{ACKNOWLEDGEMENTS}

We thank Drs Kazuhisa Iwabuchi and Akimasa Someya for invaluable help and discussion; Tomomi Ikeda and Takako Shigihara-Ikegami for technical assistance; and Drs Mark Raffeld and Masao Seto for the gifts of cell lines. We thank Katy Hale for manuscript review. This work was supported by the Project Research Program of Juntendo University School of Medicine (to LJ), the Japan Leukemia Research Fund (to YT) and the Research for Promoting Technological Seeds of the Japan Science and Technology Agency (to SK).

Supplementary Information accompanies the paper on British Journal of Cancer website (http://www.nature.com/bjc)

\section{REFERENCES}

Bao R, Lai CJ, Qu H, Wang D, Yin L, Zifcak B, Atoyan R, Wang J, Samson M, Forrester J, DellaRocca S, Xu GX, Tao X, Zhai HX, Cai X, Qian C (2009) CUDC-305, a novel synthetic HSP90 inhibitor with unique pharmacologic properties for cancer therapy. Clin Cancer Res 15: 4046-4057

Blandino G, Levine AJ, Oren M (1999) Mutant p53 gain of function: differential effects of different p53 mutants on resistance of cultured cells to chemotherapy. Oncogene 18: 477-485

Bodrug SE, Warner BJ, Bath ML, Lindeman GJ, Harris AW, Adams JM (1994) Cyclin D1 transgene impedes lymphocyte maturation and collaborates in lymphomagenesis with the myc gene. $E M B O J$ 13: 2124-2130
Brody J, Advani R (2006) Treatment of mantle cell lymphoma: current approach and future directions. Crit Rev Oncol Hematol 58: $257-265$

Chen L, Willis SN, Wei A, Smith BJ, Fletcher JI, Hinds MG, Colman PM, Day CL, Adams JM, Huang DC (2005) Differential targeting of prosurvival Bcl-2 proteins by their BH3-only ligands allows complementary apoptotic function. Mol Cell 17: 393-403

Chou TC, Talalay P (1984) Quantitative analysis of dose-effect relationships: the combined effects of multiple drugs or enzyme inhibitors. Adv Enzyme Regul 22: 27-55 
Csermely P, Schnaider T, Soti C, Prohaszka Z, Nardai G (1998) The 90-kDa molecular chaperone family: structure, function, and clinical applications. A comprehensive review. Pharmacol Ther 79: 129-168

Difeo A, Huang F, Sangodkar J, Terzo EA, Leake D, Narla G, Martignetti JA (2009) KLF6-SV1 is a novel antiapoptotic protein that targets the BH3only protein NOXA for degradation and whose inhibition extends survival in an ovarian cancer model. Cancer Res 69: 4733-4741

Döhner H, Fischer K, Bentz M, Hansen K, Benner A, Cabot G, Diehl D, Schlenk R, Coy J, Stilgenbauer S, Volkmann M, Galle PR, Poustka A, Hunstein W, Lichter P (1995) p53 gene deletion predicts for poor survival and non-response to therapy with purine analogs in chronic B-cell leukemias. Blood 85: 1580 - 1589

Donnelly AC, Mays JR, Burlison JA, Nelson JT, Vielhauer G, Holzbeierlein J, Blagg BS (2008) The design, synthesis, and evaluation of coumarin ring derivatives of the novobiocin scaffold that exhibit antiproliferative activity. J Org Chem 73: $8901-8920$

Elo MA, Kaarniranta K, Helminen HJ, Lammi MJ (2005) Hsp90 inhibitor geldanamycin increases hsp70 mRNA stabilisation but fails to activate HSF1 in cells exposed to hydrostatic pressure. Biochim Biophys Acta 1743: $115-119$

Fernàndez V, Hartmann E, Ott G, Campo E, Rosenwald A (2005) Pathogenesis of mantle-cell lymphoma: all oncogenic roads lead to dysregulation of cell cycle and DNA damage response pathways. J Clin Oncol 23: 6364-6369

Flørenes VA, Maelandsmo GM, Forus A, Andreassen A, Myklebost O, Fodstad O (1994) MDM2 gene amplification and transcript levels in human sarcomas: relationship to TP53 gene status. J Natl Cancer Inst 86: $1297-1302$

Frazier MW, He X, Wang J, Gu Z, Cleveland JL, Zambetti GP (1998) Activation of c-myc gene expression by tumor-derived p53 mutants requires a discrete C-terminal domain. Mol Cell Biol 18: 3735-3743

Gooljarsingh LT, Fernandes C, Yan K, Zhang H, Grooms M, Johanson K, Sinnamon RH, Kirkpatrick RB, Kerrigan J, Lewis T, Arnone M, King AJ, Lai Z, Copeland RA, Tummino PJ (2006) A biochemical rationale for the anticancer effects of Hsp90 inhibitors: slow, tight binding inhibition by geldanamycin and its analogues. Proc Natl Acad Sci USA 103: 7625-7630

Goy A, Bernstein SH, Kahl BS, Djulbegovic B, Robertson MJ, de Vos S, Epner E, Krishnan A, Leonard JP, Lonial S, Nasta S, O'Connor OA, Shi H, Boral AL, Fisher RI (2009) Bortezomib in patients with relapsed or refractory mantle cell lymphoma: updated time-to-event analyses of the multicenter phase 2 PINNACLE study. Ann Oncol 20: 520-525

Greiner TC, Dasgupta C, Ho VV, Weisenburger DD, Smith LM, Lynch JC, Vose JM, Fu K, Armitage JO, Braziel RM, Campo E, Delabie J, Gascoyne RD, Jaffe ES, Muller-Hermelink HK, Ott G, Rosenwald A, Staudt LM, Im MY, Karaman MW, Pike BL, Chan WC, Hacia JG (2006) Mutation and genomic deletion status of ataxia telangiectasia mutated (ATM) and p53 confer specific gene expression profiles in mantle cell lymphoma. Proc Natl Acad Sci USA 103: 2352-2357

Hartson SD, Thulasiraman V, Huang W, Whitesell L, Matts RL (1999) Molybdate inhibits Hsp90, induces structural changes in its C-terminal domain, and alters its interactions with substrates. Biochemistry 38: $3837-3849$

Hauck P, Chao BH, Litz J, Krystal GW (2009) Alterations in the Noxa/Mcl-1 axis determine sensitivity of small cell lung cancer to the $\mathrm{BH} 3$ mimetic ABT-737. Mol Cancer Ther 8: 883-892

Issacs JS, Xu W, Neckers L (2003) Heat shock protein 90 as a molecular target for cancer therapeutics. Cancer Cell 3: 213-217

Jadayel DM, Lukas J, Nacheva E, Bartkova J, Stranks G, De Schouwer PJ, Lens D, Bartek J, Dyer MJ, Kruger AR, Catovsky D (1997) Potential role for concurrent abnormalities of the cyclin D1, p16CDKN2 and $p 15 C D K N 2 B$ genes in certain B-cell non-Hodgkin's lymphomas: functional studies in a cell line (Granta 519). Leukemia 11: 64-72

Jares P, Colomer D, Campo E (2007) Genetic and molecular pathogenesis of mantle cell lymphoma: perspectives for new targeted therapeutics. Nat Rev Cancer 7: 750-762

Kabeya Y, Mizushima N, Ueno T, Yamamoto A, Kirisako T, Noda T, Kominami E, Ohsumi Y, Yoshimori T (2000) LC3, a mammalian homologue of yeast Apg8p, is localized in autophagosome membranes after processing. EMBO J 19: $5720-5728$

Kamitsuji Y, Kuroda J, Kimura S, Toyokuni S, Watanabe K, Ashihara E, Tanaka H, Yui Y, Watanabe M, Matsubara H, Mizushima Y, Hiraumi Y, Kawata E, Yoshikawa T, Maekawa T, Nakahata T, Adachi S (2008) The Bcr-Abl kinase inhibitor INNO-406 induces autophagy and different modes of cell death execution in Bcr-Abl-positive leukemias. Cell Death Differ 15: $1712-1722$
Kimura S, Ito C, Jyoko $\mathrm{N}$, Segawa $\mathrm{H}$, Kuroda J, Okada M, Adachi S, Nakahata T, Yuasa T, Filho VC, Furukawa H, Maekawa T (2005) Inhibition of leukemic cell growth by a novel anti-cancer drug (GUT-70) from Calophyllum brasiliense that acts by induction of apoptosis. Int $J$ Cancer 113: $158-165$

Kuroda J, Kimura S (2007) The role of BH3-only proteins in cancer and anti-cancer therapeutics. Cell Apoptosis Research Trends. In Zhang CV (ed) pp 1-39. Nova: New York

Lai R, McDonnell TJ, O’Connor SL, Medeiros LJ, Oudat R, Keating M, Morgan MB, Curiel TJ, Ford RJ (2002) Establishment and characterization of a new mantle cell lymphoma cell line, MINO. Leuk Res 26: 849-855

Lemasters JJ, Nieminen AL, Qian T, Trost LC, Elmore SP, Nishimura Y, Crowe RA, Cascio WE, Bradham CA, Brenner DA, Herman B (1998) The mitochondrial permeability transition in cell death: a common mechanism in necrosis, apoptosis and autophagy. Biochim Biophys Acta 1366: $177-196$

Levine B, Klionsky DJ (2004) Development by self-digestion: molecular mechanisms and biological functions of autophagy. Dev Cell 6: 463-477

Majno G, Joris I (1995) Apoptosis, oncosis, and necrosis. An overview of cell death. Am J Pathol 146: 3-15

Marcu MG, Schulte TW, Neckers L (2000) Novobiocin and related coumarins and depletion of heat shock protein 90 -dependent signaling proteins. J Natl Cancer Inst 92: 242-248

Matsui H, Asou H, Inaba T (2007) Cytokines direct the regulation of Bim mRNA stability by heat-shock cognate protein 70. Mol Cell 25: 99-112

Mei Y, Xie C, Xie W, Tian X, Li M, Wu M (2007) Noxa/Mcl-1 balance regulates susceptibility of cells to camptothecin-induced apoptosis. Neoplasia 9: $871-881$

Melo JV, Brito-Babapulle V, Foroni L, Robinson DS, Luzzatto L, Catovsky D (1986) Two new cell lines from B-prolymphocytic leukaemia: characterization by morphology, immunological markers, karyotype and Ig gene rearrangement. Int J Cancer 38: $531-538$

Mimnaugh EG, Xu W, Vos M, Yuan X, Isaacs JS, Bisht KS, Gius D, Neckers L (2004) Simultaneous inhibition of hsp 90 and the proteasome promotes protein ubiquitination, causes endoplasmic reticulum-derived cytosolic vacuolization, and enhances antitumor activity. Mol Cancer Ther 3: $551-566$

Mimnaugh EG, Xu W, Vos M, Yuan X, Neckers L (2006) Endoplasmic reticulum vacuolization and valosin-containing protein relocalization result from simultaneous hsp90 inhibition by geldanamycin and proteasome inhibition by velcade. Mol Cancer Res 4: 667-681

Muller P, Hrstka R, Coomber D, Lane DP, Vojtesek B (2008) Chaperonedependent stabilization and degradation of p53 mutants. Oncogene 27: $3371-3383$

Oda E, Ohki R, Murasawa H, Nemoto J, Shibue T, Yamashita T, Tokino T, Taniguchi T, Tanaka N (2000) Noxa, a BH3-only member of the Bcl-2 family and candidate mediator of $p 53$-induced apoptosis. Science 288: $1053-1058$

Pérez-Galán P, Roué G, Villamor N, Campo E, Colomer D (2006) The proteasome inhibitor bortezomib induces apoptosis in mantle-cell lymphoma through generation of ROS and Noxa activation independent of p53 status. Blood 107: 257-264

Pratt WB, Toft DO (2003) Regulation of signaling protein function and trafficking by the hsp90/hsp70-based chaperone machinery. Exp Biol Med 228: $111-133$

Quintanilla-Martinez L, Davies-Hill T, Fend F, Calzada-Wack J, Sorbara L, Campo E, Jaffe ES, Raffeld M (2003) Sequestration of p27Kip1 protein by cyclin D1 in typical and blastic variants of mantle cell lymphoma (MCL): implications for pathogenesis. Blood 101: $3181-3187$

Raynaud SD, Bekri S, Leroux D, Grosgeorge J, Klein B, Bastard C, Gaudray P, Simon MP (1993) Expanded range of 11q13 breakpoints with differing patterns of cyclin D1 expression in B-cell malignancies. Genes Chromosomes Cancer 8: 80-87

Robles AI, Wright MH, Gandhi B, Feis SS, Hanigan CL, Wiestner A, Varticovski L (2006) Schedule-dependent synergy between the heat shock protein 90 inhibitor 17-(dimethylaminoethylamino)-17-demethoxygeldanamycin and doxorubicin restores apoptosis to p53-mutant lymphoma cell lines. Clin Cancer Res 12: 6547-6556

Sampath J, Sun D, Kidd VJ, Grenet J, Gandhi A, Shapiro LH, Wang Q, Zambetti GP, Schuetz JD (2006) Mutant p53 cooperates with ETS and selectively up-regulates human MDR1 not MRP1. Clin Cancer Res 12: $3459-3469$

Samraj AK, Stroh C, Fischer U, Schulze-Osthoff K (2006) The tyrosine kinase Lck is a positive regulator of the mitochondrial apoptosis pathway by controlling Bak expression. Oncogene 25: 186-197 
Shen S, Zhang P, Lovchik MA, Li Y, Tang L, Chen Z, Zeng R, Ma D, Yuan J, Yu Q (2009) Cyclodepsipeptide toxin promotes the degradation of Hsp90 client proteins through chaperone-mediated autophagy. J Cell Biol 185: $629-639$

Sherr CJ, Roberts JM (1999) CDK inhibitors: positive and negative regulators of $\mathrm{G}_{1}$-phase progression. Genes Dev 13: $1501-1512$

Smit LA, Hallaert DY, Spijker R, de Goeij B, Jaspers A, Kater AP, van Oers $\mathrm{MH}$, van Noesel CJ, Eldering E (2007) Differential Noxa/Mcl-1 balance in peripheral versus lymph node chronic lymphocytic leukemia cells correlates with survival capacity. Blood 109: 1660-1668

Tse AN, Sheikh TN, Alan H, Chou TC, Schwartz GK (2009) 90-kDa heat shock protein inhibition abrogates the topoisomerase I poison-induced G2/M checkpoint in p53-null tumor cells by depleting Chk1 and Wee1. Mol Pharmacol 75: $124-133$

Tsujimoto Y, Shimizu S (2005) Another way to die: autophagic programmed cell death. Cell Death Differ 12: $1528-1534$

Van Cruchten S, Van Den Broeck W (2002) Morphological and biochemical aspects of apoptosis, oncosis and necrosis. Anat Histol Embryol 31: $214-223$

Warr MR, Shore GC (2008) Unique biology of Mcl-1: therapeutic opportunities in cancer. Curr Mol Med 8: 138-147

Willis SN, Chen L, Dewson G, Wei A, Naik E, Fletcher JI, Adams JM, Huang DC (2005) Proapoptotic Bak is sequestered by Mcl-1 and Bcl-xL, but not Bcl-2, until displaced by BH3-only proteins. Genes Dev 19: 1294-1305

Yan W, Chen X (2009) Identification of GRO1 as a critical determinant for mutant p53 gain of function. J Biol Chem 284: $12178-12187$

Zhang R, Luo D, Miao R, Bai L, Ge Q, Sessa WC, Min W (2005) Hsp90-Akt phosphorylates ASK1 and inhibits ASK1-mediated apoptosis. Oncogene 24: $3954-3963$

Zhang Y, Wang JS, Chen LL, Cheng XK, Heng FY, Wu NH, Shen YF (2004) Repression of hsp90beta gene by p53 in UV irradiation-induced apoptosis of Jurkat cells. J Biol Chem 279: 42545-42551 